\title{
Dominant transport pathways in an atmospheric blocking event
}

\author{
Enrico Ser-Giacomi, ${ }^{1}$ Ruggero Vasile, ${ }^{2}$ Irene Recuerda, ${ }^{1}$ Emilio Hernández-García, ${ }^{1}$ \\ and Cristóbal López ${ }^{1}$ \\ ${ }^{1}$ IFISC (CSIC-UIB), Instituto de Física Interdisciplinar y Sistemas Complejos, Campus Universitat de les Illes \\ Balears, E-07122 Palma de Mallorca, Spain \\ ${ }^{2}$ Ambrosys GmbH, Albert-Einstein-Str. 1-5, 14473 Potsdam, Germany
}

(Received 15 January 2015; accepted 5 August 2015; published online 19 August 2015)

\begin{abstract}
A Lagrangian flow network is constructed for the atmospheric blocking of Eastern Europe and Western Russia in summer 2010. We compute the most probable paths followed by fluid particles, which reveal the Omega-block skeleton of the event. A hierarchy of sets of highly probable paths is introduced to describe transport pathways when the most probable path alone is not representative enough. These sets of paths have the shape of narrow coherent tubes flowing close to the most probable one. Thus, even when the most probable path is not very significant in terms of its probability, it still identifies the geometry of the transport pathways. (C) 2015 AIP Publishing LLC.

[http://dx.doi.org/10.1063/1.4928704]
\end{abstract}

Eastern Europe and Western Russia experienced a strong heat wave with devastating consequences in the summer of 2010. This was due to an atmospheric blocking episode that lasted during several weeks. Despite these types of events have been well-investigated over the years, a complete understanding and prediction is still missing. In this work, we present a characterization of this flow pattern based on the study of fluid transport as a Lagrangian flow network, so that the methodology of complex networks can be applied. In particular, the most probable paths (MPPs) linking nodes of this atmospheric network reveal the dominant pathways traced by atmospheric fluid particles.

\section{INTRODUCTION}

Lagrangian analysis of transport in fluids, in particular in geophysical and time-dependent contexts, has experienced intense developments in the last decades. These can be roughly classified into three classes: Some of the approaches search for geometric objects-lines, surfaces, usually related to invariant manifolds-which bound fluid regions with different properties. ${ }^{1-3}$ In the second type of approaches, one computes different types of Lyapunov exponents and other stretching-like fields in the fluid domain. ${ }^{4-7}$ Finally, setoriented methods ${ }^{8-12}$ address directly the motions of finitesize regions.

Most of these techniques focus in identifying proper Lagrangian Coherent Structures, ${ }^{13-15}$ understood as barriers to transport or coherent regions with small fluid exchange with the surrounding medium. Much less is known about the actual routes of transport, the dominant pathways along which fluid particles travel and fluid properties are interchanged.

In principle, the pathways are simply given by trajectories starting from the desired initial conditions. This is true when the advection dynamics is represented by a deterministic dynamical system and the initial condition is precisely fixed. In many applications, however, particularly in geosciences, stochastic components are added to the motions to better represent unresolved spatial scales. ${ }^{16-18}$ Also, imprecisely stated initial conditions will develop into a divergent set of possible trajectories, because of the inherently chaotic character of advection by nearly any nontrivial fluid flow, particularly when it is time-dependent. In fact, in real experiments such as in the deployment of buoys or balloons, the trajectories of closely released objects diverge soon. ${ }^{19-21}$ The so-called spaghetti plots ${ }^{18}$ provide a visual representation of this dispersion. But they become, when many trajectories are represented, cluttered and unclear. Some types of clustering or the selection of relevant trajectories is needed to highlight which are the dominant routes among a large set of possible trajectories.

We have recently developed ${ }^{22}$ a formalism that computes, in unsteady flows, the optimal fluid paths starting at given initial conditions and also optimal paths connecting pairs of points. By optimal we refer to the paths that are more likely to be followed, in a well-defined sense made explicit below, by the fluid particles initialized in a finite neighborhood of the initial locations. By this reason, they are called most probable paths. The methodology builds on the set-oriented techniques ${ }^{8-12}$ that discretize space to provide a coarse-grained description of transport and draws analogies with network theory, ${ }^{22-26}$ for which tools to compute optimal paths in graphs are well developed. A related formalism addressing optimal paths in time-independent flows in continuous time has been developed by Metzner et $a l .{ }^{27}$ The optimal paths provide the main pathways or skeleton of the transport process in a given geographical area. Because of the implicit stochastic ingredient in the coarse-graining procedure of set-oriented methods, this methodology, at variance with other ones more tied to the theory of smooth dynamical systems, can be applied equally well to cases of deterministic transport and to strongly diffusive situations. 
In this paper, we compute optimal transport paths for the atmospheric circulation during a blocking event occurring in Summer 2010 (in particular, we focus our study for the period 20th July-30th July) over Eastern Europe and Russia. This atmospheric flow has very different temporal and spatial scales, and is much more diffusive, than the oceanic flow analyzed in Ser-Giacomi et al. ${ }^{22}$ We give a more detailed description of the methodology sketched in that reference and generalize it to extend the concept of most probable path to a hierarchy of sets of paths characterized by an increasing probability. The spatial coherence of these sets is also discussed.

The paper is organized as follows: In Sec. II, we summarize the definition and construction of the optimal pathways as most probable paths in a flow network. In Sec. III, we extend this concept to sets of highly probable paths (HPPs) and give rules to establish their significance and spatial coherence. Section IV describes the atmospheric blocking event, the data, and models we use to compute the Lagrangian trajectories and construct the flow network from them. Section V contains our results: optimal pathways for different dates and locations and also a discussion of the statistical representativeness of the optimal paths on the sets of highly probable paths. Section VI summarizes our conclusions. An Appendix applies our formalism to a simple model flow, an analytic double-gyre system, so that the properties of the optimal and highly probable paths computed for the atmospheric dynamics could be more easily understood in this simplified framework.

\section{OPTIMAL PATHS FROM LAGRANGIAN FLOW NETWORKS}

Our approach to find optimal paths in time-dependent fluid flows first represents the fluid transport dynamics as a time-dependent flow network ${ }^{26}$ and then uses graph-theory techniques to extract from it these optimal paths. Following the set-oriented methodology, ${ }^{8-12,26}$ we proceed first by a discretization of the spatial domain of interest, dividing it into $N$ non-overlapping boxes. In terms of the network-theory approach to transport, ${ }^{22,23,26}$ each of these boxes will represent a single network node. A large number of ideal fluid particles are released in each box. Under advection by a given velocity field, links between nodes are established by studying the Lagrangian trajectories of the particles exchanged among each pair of network nodes. This is conveniently done with a temporal discretization, i.e., we consider the dynamics restricted to a time interval $\left[t_{0}, t_{M}\right]$ and divide it in time steps of length $\tau, t_{l}=t_{0}+l \tau, \quad l=0,1, \ldots, M$. For each time interval $\left[t_{l-1}, t_{l}\right]$, we integrate the equations of motion of each ideal fluid particle and keep track of each trajectory. The transport dynamics will then be described by adjacency matrices $\mathbf{A}^{(l)},(l=1 \ldots M)$, in which a matrix element $\mathbf{A}_{I J}^{(l)}$ is given by the number of particles initialized at time $t_{l-1}$ in node $I$ that ends up at time $t_{l}$ in node $J$. Since the velocity field will vary in time, the adjacency matrices will depend on the time interval considered. The weighted network we build will therefore have an explicit time-dependent character and can be analyzed, for instance, using time-ordered graphs. ${ }^{22,28}$
A fundamental assumption we make is that of a Markovian dynamics, i.e., at each time interval, the ideal fluid particles are initialized with uniform density in each box, thus without keeping track of the trajectories at the previous time step. The effect of such assumption is to introduce diffusive effects in the dynamics even when the original equations of motion are fully deterministic. ${ }^{29}$ In the limit of very small boxes and very short time steps, this computational diffusion is suppressed and we approach the perfect Lagrangian motion under the given velocity field (which itself can contain diffusive or fluctuating terms).

In our network approach, spatio-temporal particle trajectories are mapped into discretized paths between the network nodes. We define an $M$-step path $\mu$ between nodes $I$ and $J$ as the ordered sequence of $(M+1)$ nodes, $\mu=\left\{I, k_{1}, \ldots\right.$, $\left.k_{M-1}, J\right\}$, crossed to reach node $J$ at time $t_{M}$ starting from node $I$ at time $t_{0}$. Under the Markovian hypothesis, we can associate a probability to each of these paths as

$$
\left(p_{I J}^{M}\right)_{\mu}=\mathbf{T}_{I k_{1}}^{(1)}\left[\prod_{l=2}^{M-1} \mathbf{T}_{k_{l-1} k_{l}}^{(l)}\right] \mathbf{T}_{k_{M-1} J}^{(M)},
$$

where

$$
\mathbf{T}_{k_{l-1} k_{l}}^{(l)}=\frac{\mathbf{A}_{k_{l-1} k_{l}}^{(l)}}{s_{\text {out }}^{(l)}\left(k_{l-1}\right)}
$$

is the probability of a fluid particle to reach node $k_{l}$ at time $t_{l}$ if it was initialized at time $t_{l-1}$ in node $k_{l-1}$, estimated as the ratio of the number of particles doing so to the total number of particles released at the initial node and time. The quantity $s_{\text {out }}^{(l)}(k)=\sum_{j} \mathbf{A}_{k j}^{(l)}$ is called out-strength of node $k$ during the $l$-th time step.

Among all possible $M$-step paths between node $I$ and $J$, the one associated with the highest probability in Eq. (1) is called the MPP and is denoted by $\eta_{I J}^{M}$. Since this path depends explicitly on the number $M$ of steps considered, it could be also named "fixed-time most probable path." Its probability is denoted by $P_{I J}^{M}=\max _{\mu}\left\{\left(p_{I J}^{M}\right)_{\mu}\right\}$. To find the MPP and its probability, we use an adaptation of the Dijkstra algorithm, ${ }^{30}$ which takes into account the layered and directed structure of our time-ordered flow graph. The simplest implementation of the algorithm would involve finding maxima by searching over the full network, which can be a computationally expensive task. This is greatly facilitated by using the concepts of accessibility and accessibility matrices. ${ }^{31}$ Thus, for given $I$ and $J$, our implementation of the algorithm consists of two main parts. In the first part, one builds the tables $\mathbf{U}_{I J}^{(l)}$ of nodes accessible from $I$ and $J$ at time step $l$, i.e., the set of nodes that can be crossed at $t=t_{l}$ coming from $I$ and proceeding towards $J$ (see Fig. 1). Technically, this is done by including in $\mathbf{U}_{I J}^{(l)}$ the nodes $k_{l}$ for which the two following conditions are satisfied:

$$
\left[\prod_{i=1}^{l} \mathbf{A}^{(i)}\right]_{I k_{l}} \neq 0 \text { and }\left[\prod_{i=l+1}^{M} \mathbf{A}^{(i)}\right]_{k_{l} J} \neq 0 .
$$

In the second part of the algorithm, one recognizes that the structure of expression (1) allows to maximize it by 
a)

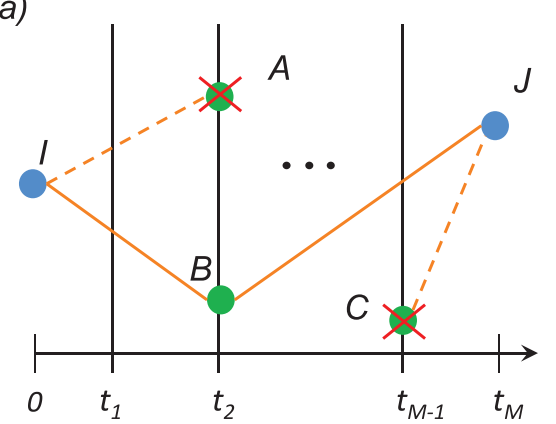

c)

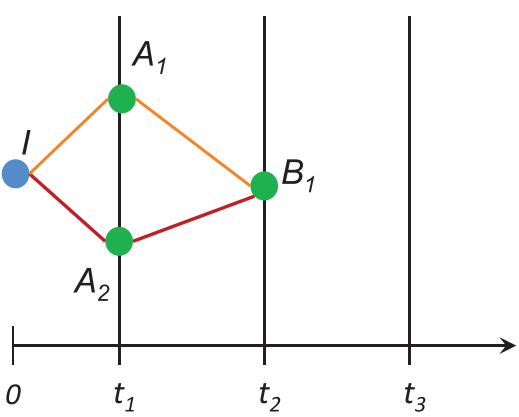

b)

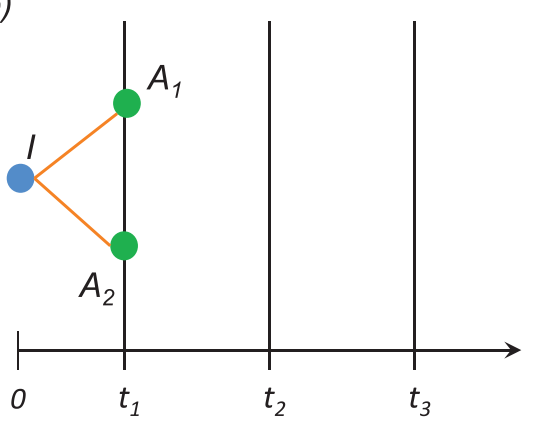

d)

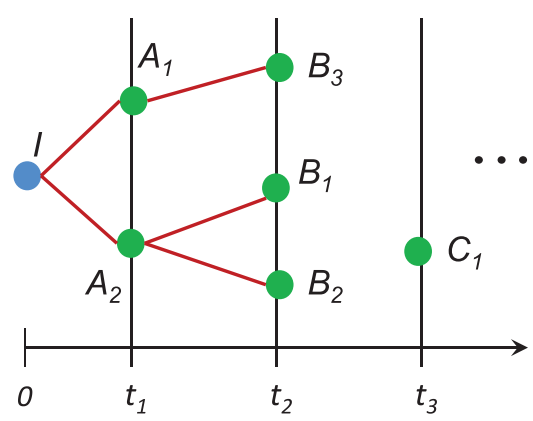

FIG. 1. Schematics of the algorithm to find the MPP of $M$ steps between $I$ and $J$. (a) First part: determination of the accessible nodes. Point $A$ is reachable from $I$ at $t=t_{2}$ but it is not possible to reach $J$ from it in the rest of the time interval. Point $C$ is not reachable from $I$ at $t=t_{M-1}$ even if $J$ can be reached from it. Point $B$ satisfies both accessibility conditions, therefore, in contrast to points $A$ and $C$, it belongs to the accessibility set and it will be considered in the calculation of the MPP. Systematic identification of all accessible nodes is done by applying the criteria in Eq. (3). The rest of the figure illustrates the recursive maximization procedure given by Eq. (4). (b) In the first time step, one assigns to the links towards the nodes $A_{1}$ and $A_{2}$ (considered to be the only ones in the accessibility set $\mathbf{U}_{I J}^{(1)}$ ) the probabilities $\mathbf{T}^{(1)}{ }_{I A_{1}}$ and $\mathbf{T}^{(1)}{ }_{I A_{2}}$, respectively. (c) For node $B_{1}$, one considers the links from $A_{1}$ and $A_{2}$, evaluates the path's probabilities $\mathbf{T}^{(1)}{ }_{I A_{1}} \mathbf{T}^{(2)}{ }_{A_{1} B_{1}}$ and $\mathbf{T}^{(1)}{ }_{I A_{2}} \mathbf{T}^{(2)}{ }_{A_{2} B_{1}}$, and selects the maximum one (in the figure corresponding to the path $I, A_{2}, B_{1}$, red lines). One repeats this for all nodes $B_{1}, B_{2}, B_{3}$ in the accessibility set $\mathbf{U}_{I J}^{(2)}$ to obtain the MPPs between $I$ and these nodes, and then the procedure can be iterated again for the accessible nodes at time $t_{3}$.

recursively maximizing over $k_{1}, k_{2}, \ldots, k_{M-1}$. This is done by finding, for each accessible node $k_{l} \in \mathbf{U}_{I J}^{(l)}$ (and only for them, without the need of scanning the remaining nodes in the full network), the highest probability $P_{I k_{l}}^{l}$ of the path connecting $I$ and $k_{l}$ and the actual path associated. For $l=1$, i.e., for the first time step, trivially we have $P_{I k_{1}}^{1}=\mathbf{T}^{(1)}{ }_{I k_{1}}$. For $l=2,3, \ldots, M-1$, we apply recursively the formula

$$
P_{I k_{l+1}}^{l+1}=\max _{k_{l}}\left(P_{I k_{l}}^{l} \mathbf{T}_{k_{l} k_{l+1}}^{(\mathbf{l}+1)}\right.
$$

until the final point $k_{M}=J$ is reached, and the maximum probability, together with the associated path, is obtained (see Fig. 1). The same procedure can then be applied to any other pair of nodes $\left(I^{\prime}, J^{\prime}\right)$.

Raising the number $M$ of steps, we observe a fast increase in the number of paths connecting two given nodes. It is thus crucial to understand how much the MPP is representative of the large set of possible paths joining two nodes. To assess this issue in a quantitative way, we introduce the following quantity:

$$
\lambda_{I J}^{M}=\frac{P_{I J}^{M}}{\sum_{\mu}\left(p_{I J}^{M}\right)_{\mu}},
$$

which determines the fraction of probability carried by the MPP with respect to the sum of probabilities of all paths connecting nodes $I$ and $J$. Note that the denominator can be simply computed as the matrix-product entry $\left(\prod_{l=1}^{M} \mathbf{T}^{(l)}\right)_{I J}$.

\section{SETS OF HIGHLY PROBABLE PATHS}

For large values of $M$, the MPP progressively loses dominance and, on average, does not carry a significantly high fraction of probability. However, the dynamics, characterized by a high number of paths connecting initial and final points, can be still described by a few of them, which together have a non-negligible probability. To see this, we can relax the definition of MPP and define a family of subsets of HPPs holding most of the probability. In our formulation, each subset $\mathcal{K}_{I J}^{M}(r, \epsilon)$ is characterized by a rank $0 \leq r \leq$ $M-1$ and a threshold parameter $0 \leq \epsilon \leq 1$. Ideally, the sets would contain all the paths whose probability is larger than $\epsilon P_{I J}^{M}$. But, since exhaustive searching of all such paths becomes computationally prohibitive except for very small $M$, the second parameter $r$ is introduced to determine the number of constraints imposed in the search for these relevant paths. Given the initial $(I)$ and final $(J)$ points, we fix $r$ nodes at intermediate times and look for paths between $I$ and $J$ made of segments, which are MPPs that connect these intermediate nodes, by using the algorithm above. Different locations and times for these $r$ intermediate nodes are scanned, and paths with probability larger than $\epsilon P_{I J}^{M}$ are retained and incorporated into the set $\mathcal{K}_{I J}^{M}(r, \epsilon)$. For $\epsilon \rightarrow 1$, independently on the rank (or for $r=0$ ), only the MPP is retained. $\mathcal{K}_{I J}^{M}(r=M-1, \epsilon)$ contains all the paths with probability larger than $\epsilon P_{I J}^{M}$. However, evaluation of these sets of HPPs can be computationally costly for high values of $r$, since the algorithm scales exponentially with $r$. 
Nevertheless, interesting results can be obtained considering already low-order HPPs, i.e., $r=1$ and $r=2$.

Once one of the subsets is computed, we can establish its significance by defining an extension of expression Eq. (5)

$$
\lambda_{I J}^{M}(r, \epsilon)=\frac{\sum_{\nu}\left(p_{I J}^{M}\right)_{\nu}}{\sum_{\mu}\left(p_{I J}^{M}\right)_{\mu}}
$$

where the sum in the numerator is over all the paths in the subset $\mathcal{K}_{I J}^{M}(r, \epsilon)$ and the one in the denominator is over all paths connecting $I$ to $J$.

Another important aspect of the sets of HPPs is to establish how close, spatially, are they with respect to the corresponding MPP. This is obtained with an average distance function. Given two generic paths between initial and final points $I$ and $J, \mu_{1}=\left\{I, k_{1}, \ldots, J\right\}$ and $\mu_{2}=\left\{I, l_{1}, \ldots, J\right\}$, we define their average distance as

$$
\mathbf{d}\left(\mu_{1}, \mu_{2}\right)=\frac{1}{M-1} \sum_{i=1}^{M-1} d\left(k_{i}, l_{i}\right)
$$

where $d\left(k_{i}, l_{i}\right)$ is a metric determining the distance between two given nodes of the network. For a geophysical transport network, the geographical distance (on the sphere) between the centers of the nodes is the most natural choice. For a given pair of nodes $(I, J)$, the average distance between the subset $\mathcal{K}_{I J}^{M}(r, \epsilon)$ and the MPP connecting them in $M$ time steps is defined as

$$
\mathcal{D}_{I J}^{M}=\frac{1}{N_{I J}^{M}} \sum_{\mu} \mathbf{d}\left(\mu, \eta_{I J}^{M}\right)
$$

where $N_{I J}^{M}$ is the number of paths $\mu$ in the subset $\mathcal{K}_{I J}^{M}(r, \epsilon)$, and the sum is extended over all paths in the subset (remember that $\eta_{I J}^{M}$ denotes the MPP). This quantity provides an estimation of how much paths in the subset deviate spatially from the correspondent MPP. A large deviation means that the probability to reach $J$ from $I$ is spatially spread in a large region and indicates furthermore the importance of considering the HPP subset instead of only the MPP. Small values of $\mathcal{D}_{I J}^{M}$ imply HPP sets with the shape of coherent narrow tubes around the MPP, so that the MPP already characterizes the spatial pathways, even if its probability is not large.

In Sections IV and V we apply the above formalism to the atmospheric flow occurring over Eastern Europe in Summer 2010. Computations of optimal paths and their sets in an analytic double-gyre system, a much simpler flow in which path properties could be more easily appreciated, are contained in the Appendix.

\section{A NETWORK OF ATMOSPHERIC FLOW OVER EASTERN EUROPE IN SUMMER 2010}

In this section, we describe the physical characteristics of the atmospheric event, the data used, and the model we employ to obtain the air particle trajectories.

\section{A. Event description}

Eastern Europe and Western Russia experienced a strong, unpredicted, heat wave during the summer of 2010.
Extreme temperatures resulted in over 50000 deaths and inflicting large economic losses to Russia. The heat wave was due to a strong atmospheric blocking that persisted over the Euro-Russian region from late June to early August. ${ }^{32}$ During July, the daily temperatures were near or above record levels and the event covered Western Russia, Belarus, Ukraine, and the Baltic nations. Physically, the origins of this heat wave were in an atmospheric block episode that produced anomalously stable anticyclonic conditions, redirecting the trajectories of migrating cyclones. Atmospheric blocks can remain in place for several days (sometimes even weeks) and are of large scale (typically larger than $2000 \mathrm{~km}$ ). In particular, the Russian block of summer 2010 was morphologically of the type known as Omega block that consists in a combination of low-high-low pressure fields with geopotential lines resembling the Greek letter $\Omega$ (see Fig.2). Omega blocks bring warmer and drier conditions to the areas that they impact and colder, wetter conditions in the upstream and downstream. ${ }^{33}$ We study the concrete period extended from 20th July to 30th July.

\section{B. Data}

Atmospheric data were provided by the National Centers for Environmental Prediction (NCEP) Climate Forecast System Reanalysis (CFSR) through the Global Forecast System (GFS). ${ }^{34}$ This reanalysis was initially completed over the 31 year period from 1979 to 2009 and extended to March 2011. Data can be obtained with a temporal resolution of $1 \mathrm{~h}$ and a spatial horizontal resolution of $0.5^{\circ} \times 0.5^{\circ}$. The spatial coverage contains a range of longitudes of $0^{\circ} \mathrm{E}$ to $359.5^{\circ} \mathrm{E}$ and latitudes of $90^{\circ} \mathrm{S}$ to $90^{\circ} \mathrm{N}$.

The variables needed as input to the Lagrangian dispersion model described in Sec. IV C include dew point temperature, geopotential height, land cover, planetary boundary layer height, pressure and pressure reduced to mean sea level, relative humidity, temperature, zonal and meridional component of the wind, vertical velocity, and water

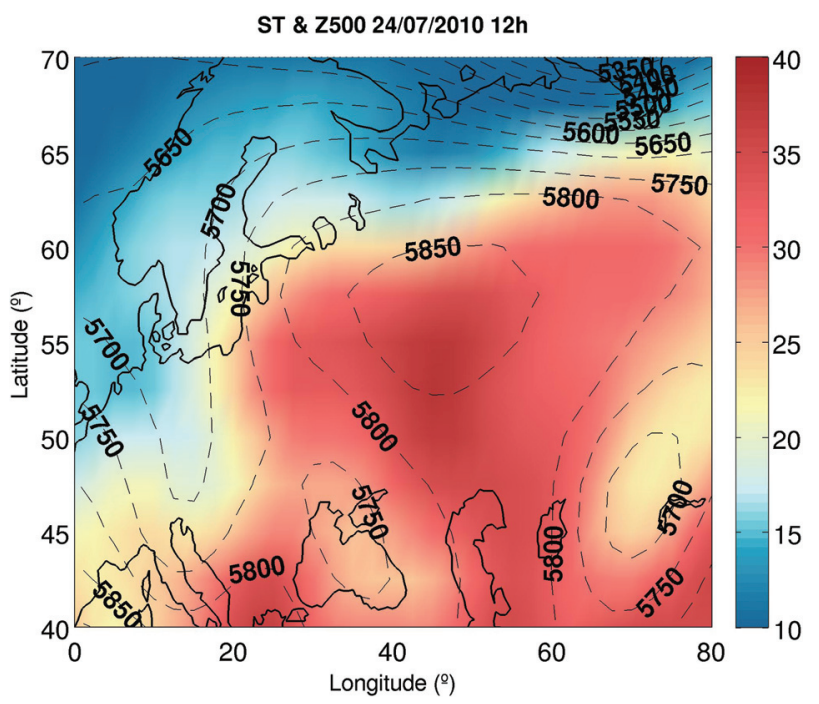

FIG. 2. Geopotential height at $500 \mathrm{hPa}$ (contours, in meters) and temperature (color code, in degree Celsius) over the region of interest, on 24th July, 12:00 UTC. 
equivalent to accumulated snow depth. All these fields are provided by CFSR data on 26 pressure levels.

\section{Lagrangian particle dispersion model FLEXPART}

As mentioned, the idea is to obtain the effective velocity field felt by any fluid particle. Then, the Lagrangian dispersion model will integrate it to provide the threedimensional positions of the particle at every time step as output.

The numerical model used to integrate particle velocities and obtain trajectories is the Lagrangian particle dispersion model FLEXPART version 8.2. ${ }^{35,36}$ FLEXPART simulates the long-range and mesoscale transport, diffusion, dry and wet deposition, and radioactive decay of tracers released from point, line, area, or volume sources. It most commonly uses meteorological input fields from the numerical weather prediction model of the European Centre for Medium-Range Weather Forecasts (ECMWF) and the GFS from NCEP (the one used in our study). Trajectories are produced by integrating the equation (the input velocity data are interpolated on the present particle position)

$$
\frac{d \mathbf{X}}{d t}=\mathbf{v}(\mathbf{X}(t))
$$

with $t$ being time, $\mathbf{X}$ the vector position of the air particle, and $\mathbf{v}=\overline{\mathbf{v}}+\mathbf{v}^{\mathbf{t}}+\mathbf{v}^{\mathbf{m}}$ is the wind vector. FLEXPART takes the grid scale wind $\overline{\mathbf{v}}$ from the CFSR but complements it with stochastic components $\mathbf{v}^{\mathbf{t}}$ and $\mathbf{v}^{\mathbf{m}}$ to better simulate the unresolved turbulent processes occurring at small scales. The turbulent wind fluctuations $\mathbf{v}^{\mathbf{t}}$ are parametrized by assuming a Markov process via a Langevin equation, and the mesoscale wind fluctuations $\mathbf{v}^{\mathbf{m}}$ are implemented also via an independent Langevin equation by assuming that the variance of the wind at the grid scale provides information on the subgrid variance. Variables entering the parametrizations are obtained from the meteorological CFSR fields. For additional details, we refer to Stohl et al. ${ }^{35,36}$

\section{Network construction}

We focus our analysis on the domain in between $0^{\circ} \mathrm{E}-80^{\circ} \mathrm{E}$ and $40^{\circ} \mathrm{N}-70^{\circ} \mathrm{N}$. In order to define the nodes of the network, we discretize this region in 626 equal-area boxes using a sinusoidal projection. The latitudinal extension of each node-box is $1.5^{\circ}$, and the longitudinal one varies depending on the latitude (see Fig. 3). The area of each box is $27722 \mathrm{~km}^{2}$, so that the typical horizontal size is of the order of $166.5 \mathrm{~km}$. This is a moderate coarse-graining of the resolution $\left(0.5^{\circ} \times 0.5^{\circ}\right)$ of the NCEP data used for particle integration. We take $\tau=12 \mathrm{~h}$ as time discretization, which is enough to follow the dynamics of the blocking event. It has been shown in an oceanic flow network ${ }^{22}$ that the value of $\tau$ has a minor influence on optimal paths, being more important the total time-interval considered $M \tau$. We uniformly fill each node with 800 ideal fluid particles releasing them at $5000 \mathrm{~m}$ of height, a representative level in the middle troposphere. FLEXPART trajectories are fully three-dimensional, but by initializing at each time-step particles in a single

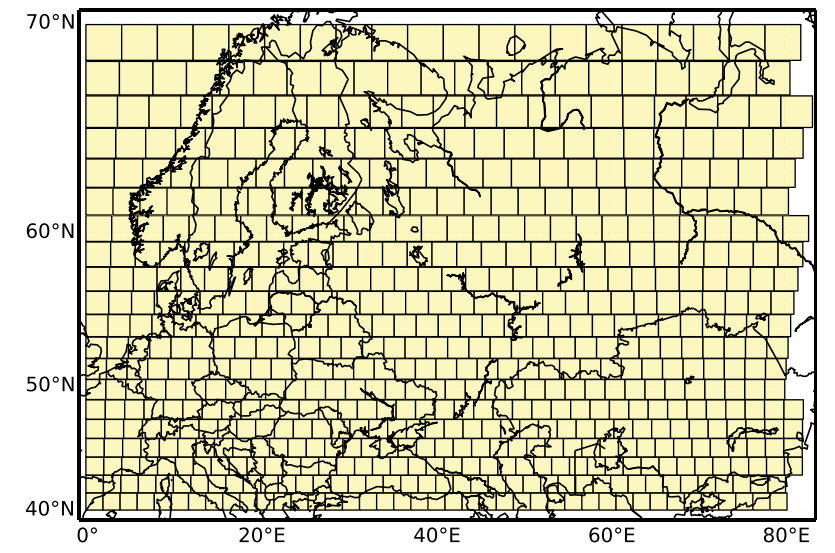

FIG. 3. The geographical domain considered and the discretization grid defining the nodes of our flow network.

layer, we are effectively neglecting the vertical dispersion (which is of the order of $800 \mathrm{~m}$ in the $\tau=12 \mathrm{~h}$ time step) and focussing on the pathways of large scale horizontal transport. Fully three-dimensional flow networks will be the subject of future work.

\section{RESULTS}

\section{A. Optimal paths}

Equipped with the tools developed above, we can now compute pathways of transport during the atmospheric event described in Sec. IV. Figure 4(a) shows all the optimal paths leaving a node in the Scandinavian Peninsula at July 25 and arriving to all nodes that are reached in $M=9$ steps (i.e., 4.5 days). The graphical representation joins with maximal arcs the center of the grid boxes identified as pertaining to the MPP. The actual particle trajectories between two consecutive boxes are not necessarily such arcs. The paths are colored according to their probability value $P_{I J}^{M}$. The MPPs with highest probability (reddish colors) follow a dominant anticyclonic (i.e., clockwise) route bordering the high pressure region (see Fig. 2, but note that this is at a particular time, whereas the trajectory plots span a range of dates of more than four days) without penetrating it. There is also a branch of MPPs with much smaller probabilities (yellow and bluish colors) that are entrained southward by a cyclonic circulation.

Despite the persistent character of the Eulerian block configuration, sets of Lagrangian trajectories become highly variable in time. See, for example, the set of MPPs starting from the same initial location but five days earlier (Fig. 4(b)). The southward cyclonic branch is now absent, all MPPs following initially the anticyclonic gyre. Remarkably, the set of trajectories bifurcates into two branches when approaching what seems to be a strong hyperbolic structure close to $40^{\circ} \mathrm{N} 60^{\circ} \mathrm{E}$. A hint of the presence of second hyperbolic structure is visible at the end of the westward branch, close to $50^{\circ} \mathrm{N} 30^{\circ} \mathrm{E}$. Figure 5 displays additional MPPs starting also at 20th July, but initialized inside the main anticyclonic region of the blocking, and in two low-pressure regions flanking it. Fig. 5(a) clearly shows the main anticyclonic circulation, highlighting also the escape routes from 

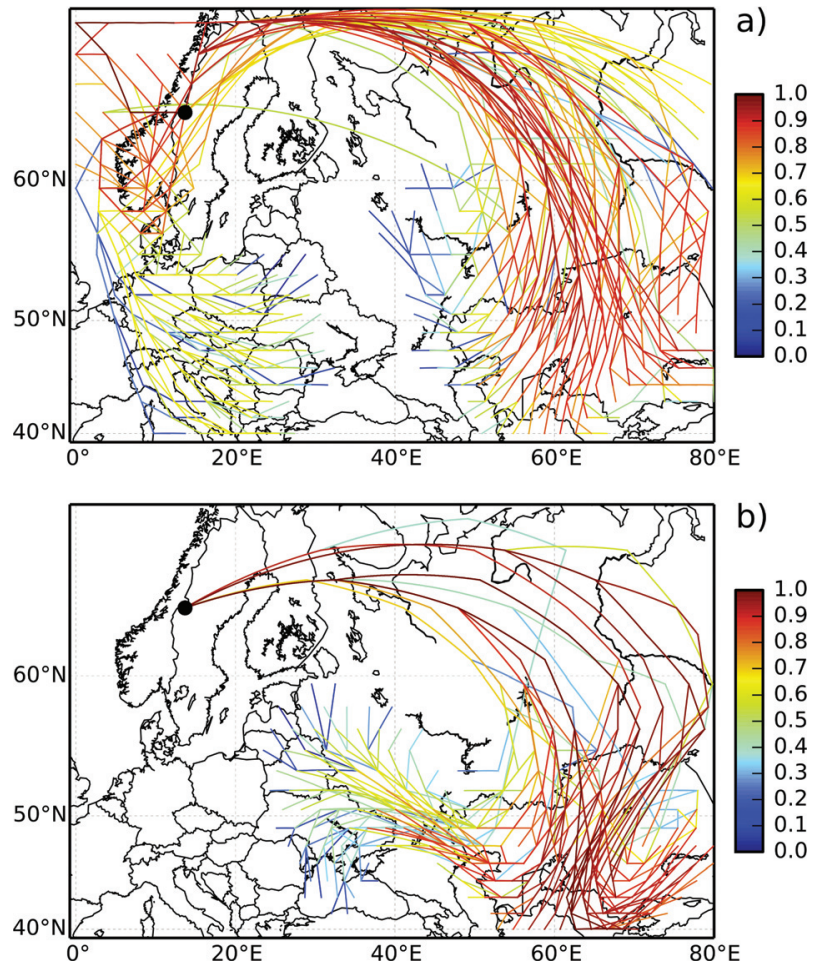

FIG. 4. Paths of $\mathrm{M}=9$ steps of $\tau=12 \mathrm{~h}$ in our flow network with starting date July 25, 2010 (panel a)) and July 20, 2010 (panel b)) represented as straight segments (in fact, maximal arcs on the Earth sphere) joining the path nodes. MPPs originating from a single node (black circle) and ending in all accessible nodes. Color gives the $P_{I J}^{M}$ value of the paths in a normalized log-scale between the minimum value (deep blue) and the maximum (dark red). Panel (a): probabilities ranging from $10^{-3}$ to $10^{-14}$. Panel (b): probabilities ranging from $10^{-3}$ to $10^{-15}$.

the high-pressure zone, associated with the hyperbolic regions described above. Panels (b) and (c) show the cyclonic circulations at each side of the high, in a characteristic Omega-blocking configuration. The compactness of the trajectories inside the eastern low-pressure area is remarkable, which forms a very localized and coherent set with practically no escape in the displayed 4.5 days time-interval.

We stress that the plots in Figs. 4 and 5 are different from spaghetti plots for which many available trajectories are plotted from different or related initial conditions. For our set of particles, this will give 800 trajectories emanating from each box. Here, we are plotting just one path, the MPP, for each initial and final box pairs, which strongly limits the number of paths from each box but, as we will see more thoroughly, it is still representative of the trajectories of many released particles.

\section{B. Relevance of the MPPs}

The range of colors in Figs. 4 and 5 indicates that, given an initial box, not all MPPs leading to different locations are equally probable. This is quantified by the probability $P_{I J}^{M}$, which gives a weight to each MPP. Indeed, $P_{I J}^{M}$ takes a very large range of values. Figure 6 shows a ranking plot in which the values of all MPPs of a given $M$ and started at a particular date are plotted in decreasing order. We see a huge spread on the values of $P_{I J}^{M}$. Very low probability values arise because of the exponential explosion of the number of paths
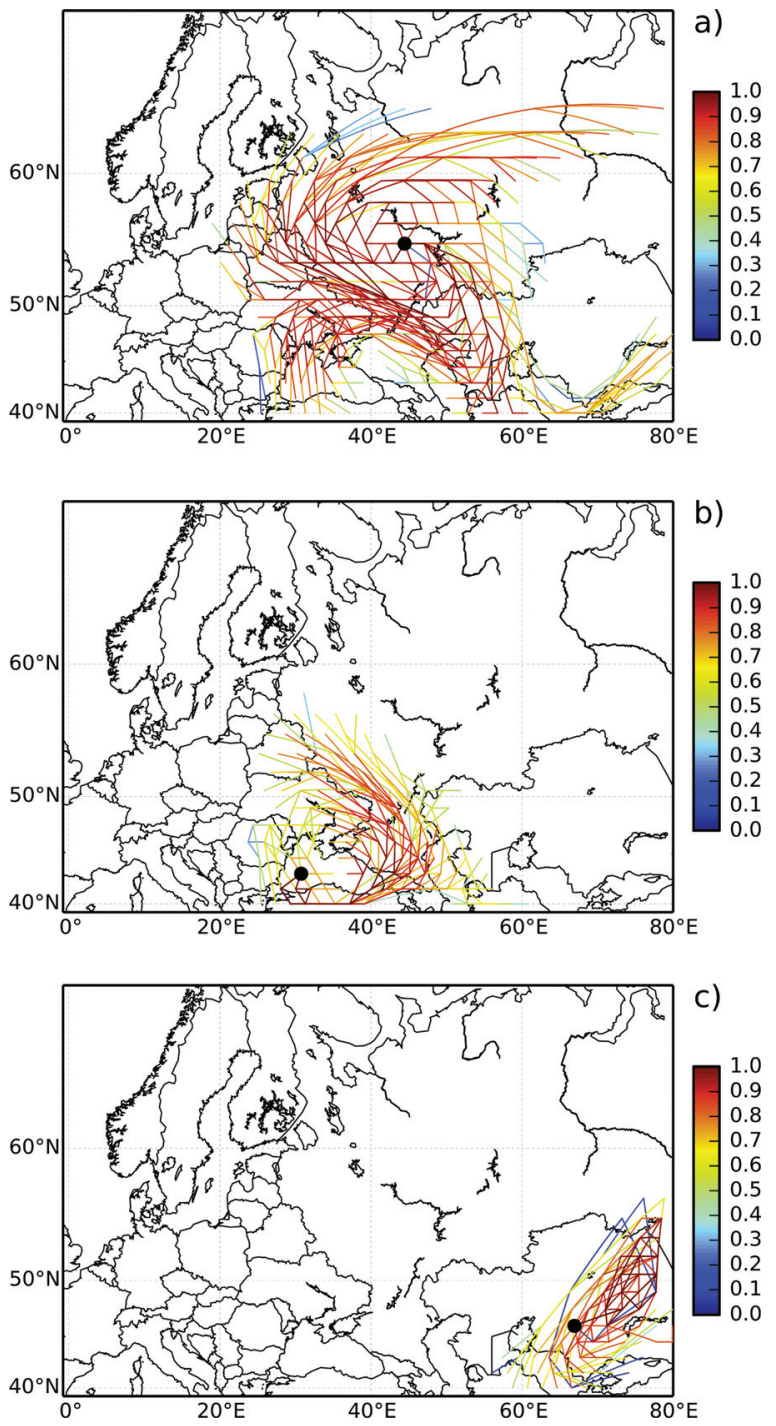

FIG. 5. Optimal paths of 9 steps of $\tau=12 \mathrm{~h}$ with starting date July 20, 2010, entrained in the high- and in the two low-pressure areas of the blocking. Same coloring scheme as in Fig. 4. Panel (a): probabilities ranging from $10^{-3}$ to $10^{-16}$. Panel (b): probabilities ranging from $10^{-2}$ to $10^{-16}$. Panel (c): probabilities ranging from $10^{-3}$ to $10^{-13}$.

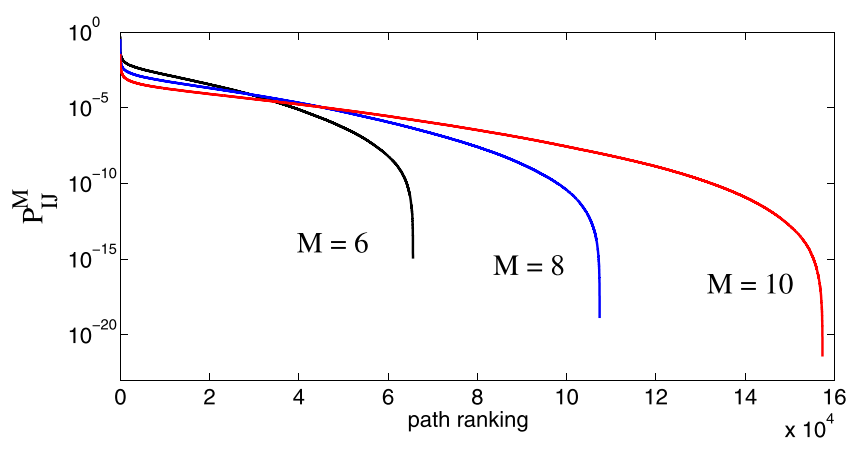

FIG. 6. Ranking plot in which the $P_{I J}^{M}$ values of all MPPs obtained for $M=6,8$, and 10 starting on July 25 th in the whole area are plotted in decreasing order. The range of probability values of the MPPs can be read from the vertical axis (from a few percent to $10^{-15}$ for $M=6$ or to less than $10^{-20}$ for $M=10$ ). The total number of optimal paths can also be read-off from the horizontal axis. 

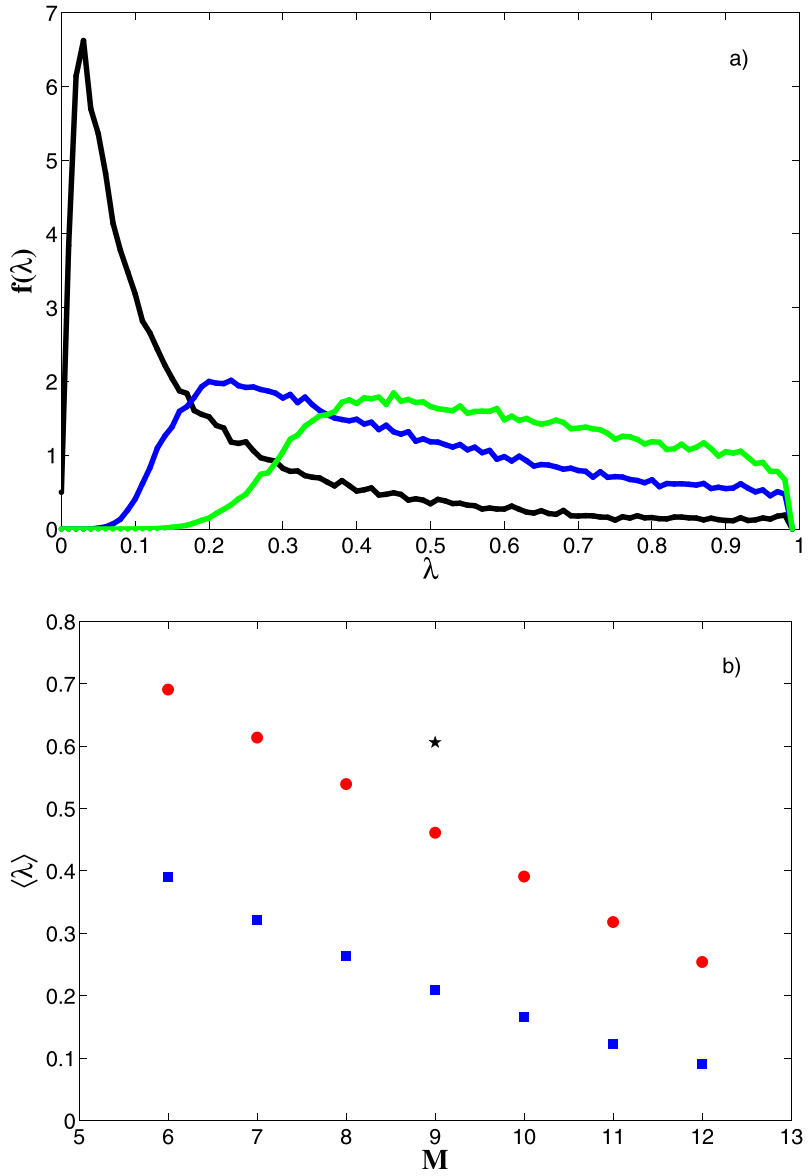

FIG. 7. (a) Normalized probability density $f(\lambda)$ of the merit figure $\lambda_{I J}^{M}(r, \epsilon)$ of paths started on July 20, 2010, for $\mathrm{M}=9$ and $\epsilon=0.1$, with $r=0$ (only the MPPs, left, black curve), $r=1$ (middle blue curve), and $r=2$ (right green curve). Statistics is compiled from all (I,J) pairs. (b) Mean value of the $\lambda_{I J}^{M}(r, \epsilon)$ distributions (paths' starting date July 25) as a function of the number of time steps $M$ for $r=0$ (only MPPs, blue squares), $r=1$ (red circles), and $r=2$ (single black star).

between two nodes with increasing $M$. Given these low values of $P_{I J}^{M}$ except for the smallest values of $M$, one should ask how representative are the MPPs for the full set of paths. Figure 7(a) shows distributions of the parameter $\lambda_{I J}^{M}(r, \epsilon)$

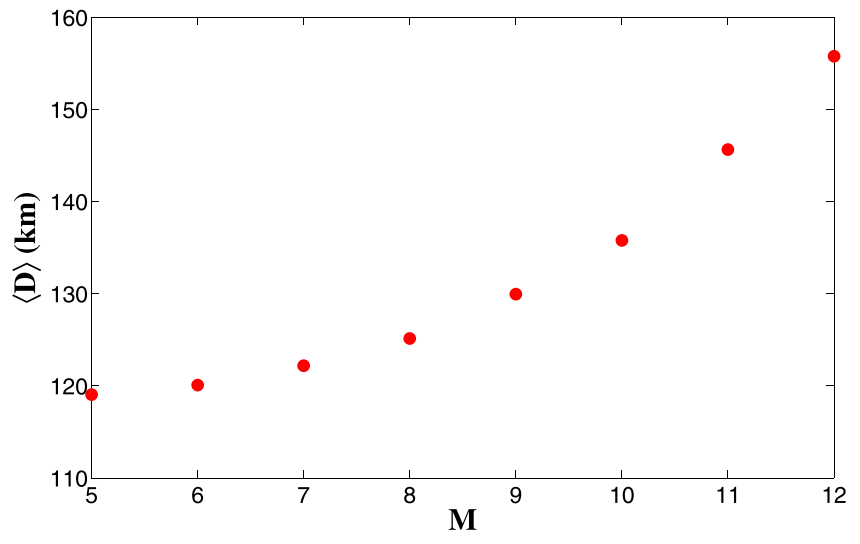

FIG. 9. Plot of the mean distance $\mathcal{D}_{I J}^{M}$ (Eq. (8)) as a function of $M$ for $r=1$ and $\epsilon=0.1$. The quantity is further averaged over all the HPPs starting on July 25 . Units are in kilometers.

giving the relative importance of the different types of paths. We see that $\lambda$-values are small when considering only the MPPs $(r=0)$, but the distributions shift towards higher values for paths sets of increasing $r$. Figure 7(b) gives mean values of the $\lambda$ distributions. They decrease with $M$, reflecting the lack of representativeness of the smallest sets of paths for large $M$. However, already for $r=1$, the set of HPPs has a mean value higher than 0.5 for a relevant range of time steps.

Thus, for the values of $M$ and $\epsilon$ discussed here, the set of HPPs with $r=1$ seems to be rich enough to represent the transport pathways. But how different is the geometry of the different paths in this HPP set? And how different is it from the MPPs? We plot in Fig. 8 examples of all HPPs with $r=1$ and $\epsilon=0$ for particular $(I, J)$ values and dates. In all the cases, the sets remain coherent and narrow tubes of trajectories, and define roughly the same pathway as the MPP.

A quantification of the width of the tubes can be done with the distance measure $\mathcal{D}_{I J}^{M}$ in Eq. (8). An average of it over pairs of locations is shown in Fig. 9. Although the tube width increases with $M$, it remains always below the typical linear box size of approximately $166.5 \mathrm{~km}$ (see Sec. IV D), indicating that the tubes remain narrow. Thus, we conclude that, despite the decreasing probability of the MPPs for
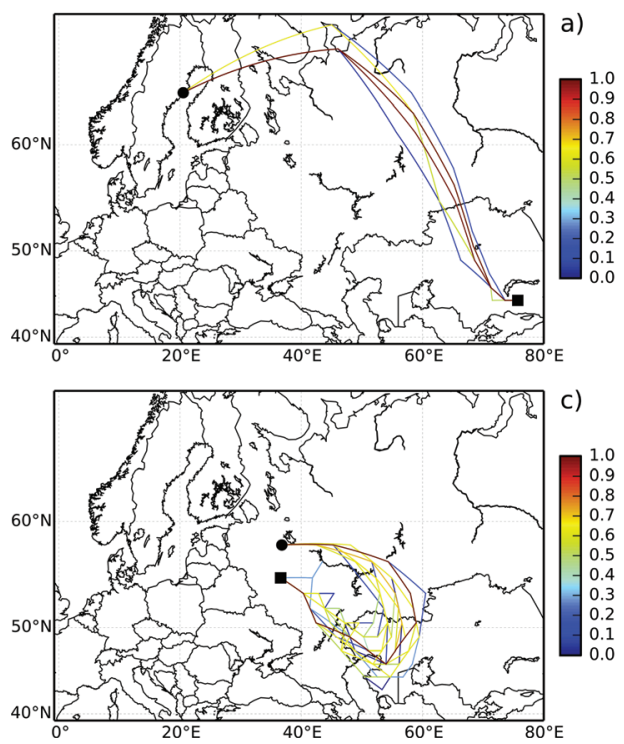
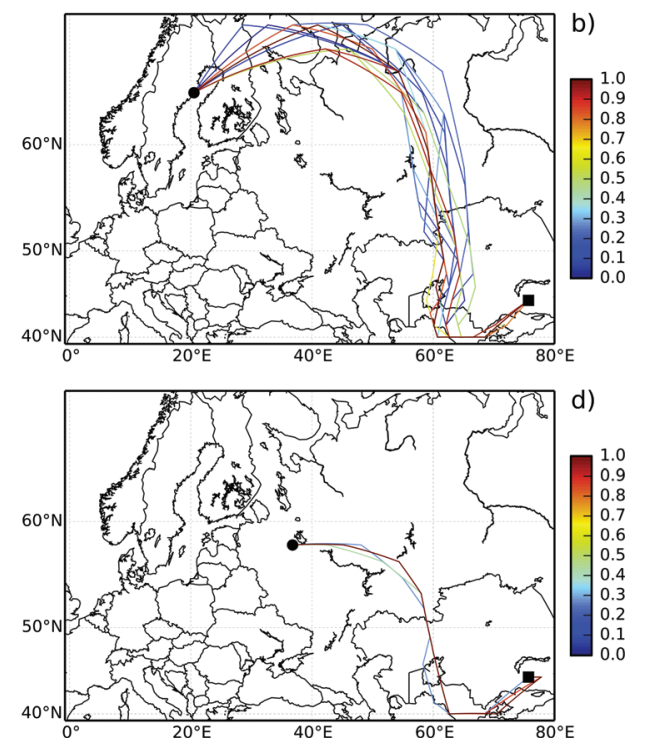

FIG. 8. All paths in $\mathcal{K}_{I J}^{M}(r=1, \epsilon=$ 0.1 ) for different $I, J$, initial point $I$ marked by a circle and final point $J$ marked by a square. The color bar gives in logarithmic scale values ranging from the maximum one $P_{I J}^{M}$ (dark red), corresponding to the MPP, to the minimum of $0.1 P_{I J}^{M}$. Panel (a): $M=8$ steps, with starting date July 25, 2011; $P_{I J}^{M}=7.8 \times 10^{-5}$. Panel (b): $M=12$ steps, with starting date July 25,2011 ; $P_{I J}^{M}=2.7 \times 10^{-5}$. Panel (c): $M=11$ steps, with starting date July 20, 2011; $P_{I J}^{M}=7.4 \times 10^{-7}$. Panel (d): $M=11$ steps, with starting date July 20, 2011; $P_{I J}^{M}=1.5 \times 10^{-7}$. 

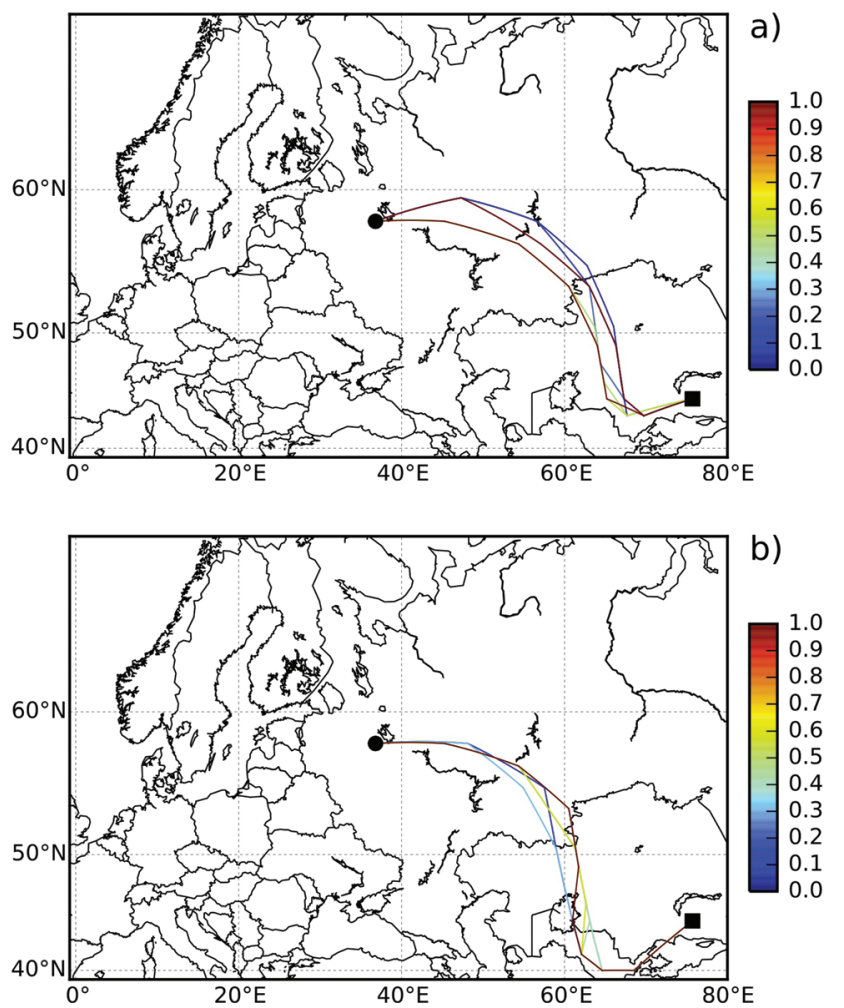

FIG. 10. All paths in $\mathcal{K}_{I J}^{M}(r=1, \epsilon=0.1)$ for the same $I, J$ and starting date as in Fig. 8(d). Same coloring scheme as in Fig. 8. Panel (a) $M=7$ steps; $P_{I J}^{M}=2.2 \times 10^{-5}$. (b) $M=9$ steps; $P_{I J}^{M}=2.3 \times 10^{-4}$.

increasing $M$, they remain good indicators of the dominant pathways in the transport network.

As a final description of properties of the dominant transport paths, we present in Figure 10 (compare with Fig. 8(d)) an example on how the MPP and the HPPs between a fixed pair of nodes change when considering different values of $M$, defining the temporal interval. Typically, the probability of the MPP shows a maximum at some intermediate values of $M$ in between shorter values of $M$ for which very few particles connect the two nodes and larger values of $M$ for which the increasing number of factors smaller than one in the product (1) defining $\left(p_{I J}^{M}\right)_{\mu}$ makes this quantity to decrease again until vanishing. For the example shown in Figs. 8(d) and 10, the value of $M$ giving the maximum $P_{I J}^{M}$ is around $M \approx 9$, i.e., $M \tau=4.5$ days. Note that the HPP trajectories change length but keep a similar shape in the range of $M$ considered, indicating that in this time interval, the blocking atmospheric structures evolve slowly.

\section{CONCLUSIONS}

We have introduced MPPs and sets of HPPs as tools to visualize and analyze dominant pathways in geophysical flows. We have computed them for an atmospheric blocking event involving Eastern Europe and Western Russia. The computed optimal paths give a Lagrangian view of the Omega-block configuration, with a central anti-cyclonic circulation flanked by two cyclonic ones. Moreover, they give additional insight on it, such as the variability of the dominant pathways, and the identification of escaping and trapping regions. The statistical significance of single MPPs decreases with the time interval considered, but we find always that the MPPs remain representative of the spatial geometry of the pathways, in the sense that the sets of HPPs are coherent narrow tubes providing transport paths always close to the optimal path. This spatial coherence of transport between pairs of locations was already noticed in an ocean flow, ${ }^{22}$ and it is also present in the model flow discussed in the Appendix. Then, it seems to be a general characteristic of flow networks.

\section{ACKNOWLEDGMENTS}

We acknowledge financial support from FEDER and MINECO (Spain) through the ESCOLA (Grant No. CTM2012-39025-C02-01) and INTENSE@COSYP (Grant No. FIS2012-30634) projects and from the European Commission Marie-Curie ITN program (FP7-320 PEOPLE2011-ITN) through the LINC project (Grant No. 289447).

\section{APPENDIX: OPTIMAL PATHS IN A SIMPLE MODEL SYSTEM}

In this Appendix, we display optimal paths and sets of optimal paths for an analytic model flow, the double-gyre. See, for example, Refs. 37 and 38 for basic properties of this system and computations of its Lagrangian coherent structures and Lyapunov fields. Because of the simplicity of this flow as compared with the atmospheric situation studied in the main text, characteristics of the optimal paths could be appreciated more easily.

The double-gyre is a two-dimensional time-periodic flow defined in the rectangular region of the plane $\mathbf{x}=(x, y) \in[0,2] \times[0,1]$. It is described by the stream function

$$
\psi(x, y, t)=A \sin (\pi f(x, t)) \sin (\pi y),
$$

with

$$
\begin{gathered}
f(x, t)=a(t) x^{2}+b(t) x, \\
a(t)=\gamma \sin (\omega t), \\
b(t)=1-2 \gamma \sin (\omega t) .
\end{gathered}
$$

From these expressions, the velocity field is

$$
\begin{array}{r}
\dot{x}=-\frac{\partial \psi}{\partial y}=-\pi A \sin (\pi f(x, t)) \cos (\pi y), \\
\dot{y}=\frac{\partial \psi}{\partial x}=\pi A \cos (\pi f(x, t)) \sin (\pi y) \frac{\partial f(x, t)}{\partial x} .
\end{array}
$$

For $\gamma=0$, this flow is steady. Ideal fluid particles follow very simple trajectories: they rotate following closed streamlines, clockwise in the left half of the rectangle and counterclockwise in the right one. The central streamline $x=1$, a heteroclinic connection between the hyperbolic point at $(1,1)$ and the one at $(1,0)$, acts as a separatrix between the two regions. When $\gamma>0$, more complex behavior including 


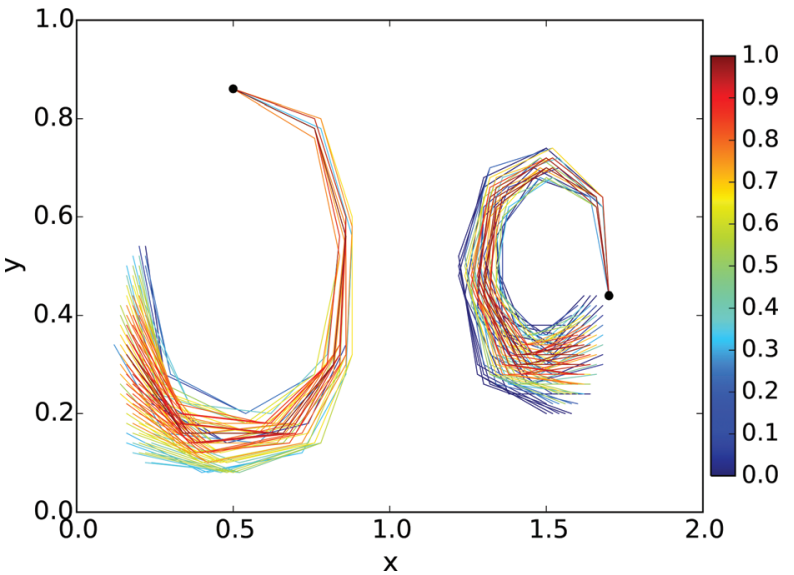

FIG. 11. Double-gyre 6-step paths in the steady case $\gamma=0$, from two different starting nodes (the black circles) to all the accessible destinations. The network nodes pertaining to each path are joined by straight line segments colored according to the path probability. The color code is logarithmic in the full probability range, which is $\left[0.054,7.73 \times 10^{-10}\right]$ for the left node (there is a total of 94 paths emanating from it) and $\left[0.0234,10^{-7}\right]$ for the right one (87 paths).

chaotic trajectories arises. The periodic perturbation breaks the separatrix, so that now some interchange of fluid is possible between the left and the right parts of the rectangle. The geometric structures involved in this interchange have been studied with a variety of techniques, ${ }^{37,38}$ but the framework of optimal paths developed in this paper seems quite natural for this purpose.

We take the parameters $A=0.1$ and $\omega=2 \pi / 5$ and compute paths in our network framework for two qualitatively different situations, namely, the steady case $\gamma=0$, and the periodically perturbed case (of period $2 \pi / \omega=5$ ) with $\gamma=0.3$. We discretize the fluid domain into $100 \times 50=5000$ square boxes, defining the nodes in our flow network, and compute the adjacency matrices $\mathbf{A}^{(l)}, l=1 \ldots M$, by releasing 400 particles from each of the boxes. In all the cases shown below, we compute paths of $M=6$ steps of duration $\tau=1$, starting at $t_{0}=0$.

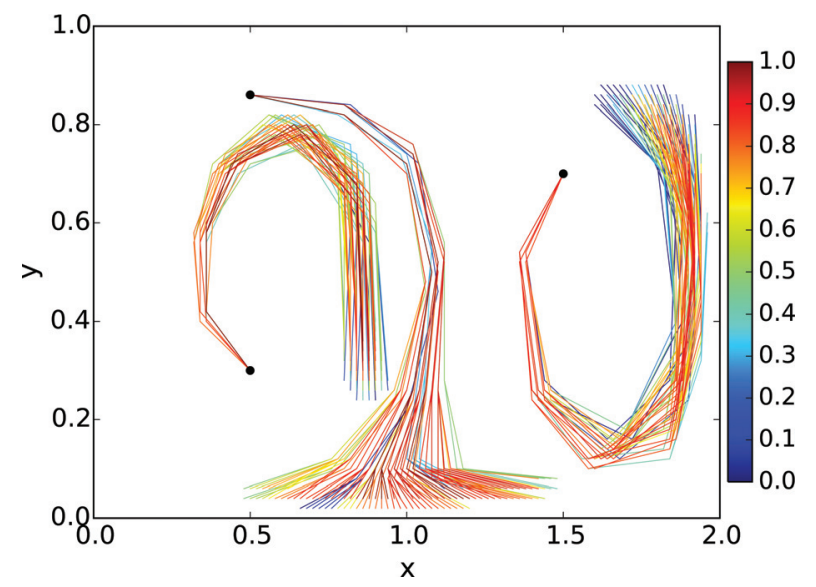

FIG. 12. 6-step paths for the periodically perturbed double gyre at $\gamma=0.3$, from three different starting nodes (black circles) to all the accessible destinations. Color coding as in Fig. 11, with probability ranges that are $\left[0.0327,3.355 \times 10^{-7}\right]$ (bottom-left node, 66 paths), $\left[0.0245,1.879 \times 10^{-9}\right]$ (top-left node, 108 paths), and $\left[0.0128,1.335 \times 10^{-8}\right]$ (right node, 106 paths).

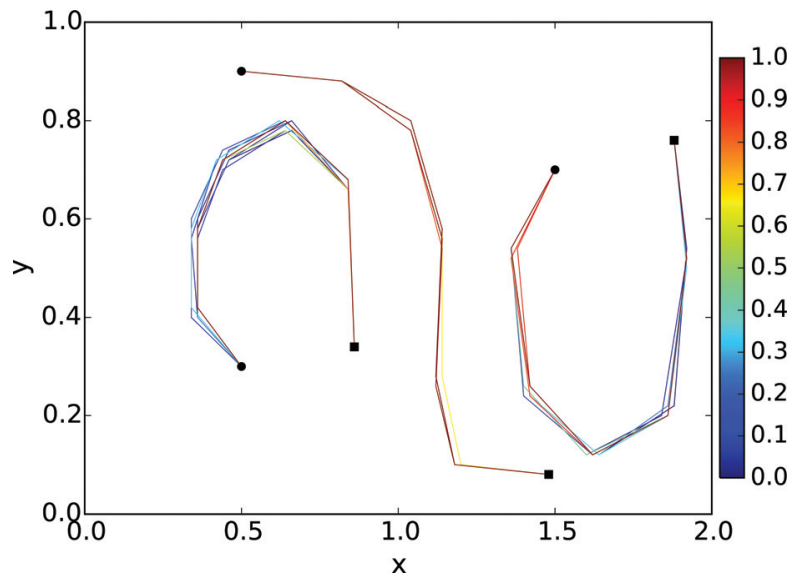

FIG. 13. Three sets of HPPs in the double gyre for $\gamma=0.3$. We show all HPPs in $\mathcal{K}_{I J}^{6}(r=1, \epsilon=0.05)$, with starting nodes $I$ at the black circles and destinations $J$ at the black squares. Color coding as in Figs. 11 and 12, with probability ranges [0.0282,0.00208] (bottom-left initial node, 12 paths), $[0.0321,0.00179]$ (top-left initial node, 7 paths), and $[0.0128,0.000946]$ (right initial node, 10 paths).

Figure 11 considers the steady flow $(\gamma=0)$ and shows all optimal paths emanating from two particular initial nodes and reaching all nodes accessible from them after the 6 steps. We see the general clockwise and anticlockwise circulations at each side of the separatrix. The two halves of the domain remain isolated. Note that the paths are different from the closed streamlines. This is so because the discretization of the fluid domain into finite boxes, together with the Markov assumption, introduces a stochastic component equivalent to an effective diffusivity ${ }^{29}$ and leads to dispersion of the particles starting from a single node. In our atmospheric velocity flow, there were in addition explicit stochastic terms modeling turbulent diffusion and mesoscale fluctuations. Note also that, as in the atmospheric case, a huge range of values of $P_{I J}^{M}$ is present.

Figure 12 shows optimal paths for the periodically perturbed flow $(\gamma=0.3)$. The general clockwise and counterclockwise rotations still remain, but now there are pathways connecting the two halves of the domain. Note the strong divergence of close pathways when they approach the hyperbolic region at the bottom of the domain, and note how is this geometric structure what allows transport of fluid between the two regions that were isolated in the steady case.

In Fig. 13, we display sets of HPPs between three pairs of nodes at $\gamma=0.3$. More specifically, we compute the paths obtained with $r=1$ and a probability larger than $5 \%$ of the $P_{I J}^{M}$ for these pairs of nodes (i.e., the paths in the set $\left.\mathcal{K}_{I J}^{6}(r=1, \epsilon=0.05)\right)$. The HPPs arrange in very narrow tubes around the MPP, which is the same behavior observed in the atmospheric paths and also in ocean calculations. ${ }^{22}$ The central path in Fig. 13 clearly identifies the pathway followed by particles to connect the left and right regions, using the "opening" around the hyperbolic region at the top of the domain.

${ }^{1}$ A. M. Mancho, D. Small, and S. Wiggins, "A tutorial on dynamical systems concepts applied to Lagrangian transport in oceanic flows defined as finite time data sets: Theoretical and computational issues," Phys. Rep. 437, 55-124 (2006). 
${ }^{2}$ G. Haller and F. J. Beron-Vera, "Geodesic theory of transport barriers in two-dimensional flows," Physica D 241, 1680-1702 (2012).

${ }^{3}$ S. Balasuriya, "Explicit invariant manifolds and specialised trajectories in a class of unsteady flows," Phys. Fluids 24, 127101 (2012).

${ }^{4} \mathrm{G}$. Haller, "Distinguished material surfaces and coherent structure in three-dimensional fluid flows," Physica D 149, 248-277 (2001).

${ }^{5}$ B. Joseph and B. Legras, "Relation between kinematic boundaries, stirring, and barriers for the antartcic polar vortex," J. Atmos. Sci. 59, 1198-1212 (2002).

${ }^{6}$ F. d'Ovidio, V. Fernández, E. Hernandez-García, and C. López, "Mixing structures in the Mediterranean Sea from finite-size Lyapunov exponents," Geophys. Res. Lett. 31, L17203, doi:10.1029/2004GL020328 (2004).

${ }^{7}$ A. M. Mancho, S. Wiggins, J. Curbelo, and C. Mendoza, "Lagrangian descriptors: A method for revealing phase space structures of general time dependent dynamical systems," Commun. Nonlinear Sci. Numer. Simul. 18, 3530-3557 (2013).

${ }^{8}$ G. Froyland and M. Dellnitz, "Detecting and locating near-optimal almostinvariant sets and cycles," SIAM J. Sci. Comput. 24, 1839-1863 (2003).

${ }^{9}$ M. Dellnitz, G. Froyland, C. Horenkamp, K. Padberg-Gehle, and A. Sen Gupta, "Seasonal variability of the subpolar gyres in the Southern Ocean: A numerical investigation based on transfer operators," Nonlinear Process. Geophys. 16, 655-663 (2009).

${ }^{10}$ G. Froyland, N. Santitissadeekorn, and A. Monahan, "Transport in timedependent dynamical systems: Finite-time coherent sets," Chaos 20, 043116 (2010).

${ }^{11}$ Z. Levnajić and I. Mezić, "Ergodic theory and visualization. I. Mesochronic plots for visualization of ergodic partition and invariant sets," Chaos 20, 033114 (2010).

${ }^{12}$ G. Froyland, C. Horenkamp, V. Rossi, N. Santitissadeekorn, and A. S. Gupta, "Three-dimensional characterization and tracking of an Agulhas Ring," Ocean Modell. 52-53, 69-75 (2012).

${ }^{13} \mathrm{G}$. Haller and G. Yuan, "Lagrangian coherent structures and mixing in two-dimensional turbulence," Physica D 147, 352-370 (2000).

${ }^{14}$ T. Peacock and J. Dabiri, "Introduction to Focus Issue: Lagrangian coherent structures," Chaos 20, 017501 (2010).

${ }^{15}$ G. Haller, "Lagrangian coherent structures," Annu. Rev. Fluid Mech. 47, 137-162 (2015).

${ }^{16} \mathrm{D}$. J. Thomson, "Criteria for the selection of stochastic models of particle trajectories in turbulent flows," J. Fluid Mech. 180, 529-556 (1987).

${ }^{17}$ A. Stohl, "Computation, accuracy and applications of trajectories-A review and bibliography," Atmos. Environ. 32, 947-966 (1998).

${ }^{18}$ M. Veneziani, A. Griffa, A. M. Reynolds, and A. J. Mariano, "Oceanic turbulence and stochastic models from subsurface Lagrangian data for the Northwest Atlantic ocean,” J. Phys. Oceanogr. 34, 1884-1906 (2004).

${ }^{19}$ B. Trounday, L. Perthuis, S. Strebelle, J. D. Farrara, and C. R. Mechoso, "Dispersion properties of the flow in the southern stratosphere during winter and spring," J. Geophys. Res.: Atmos. 100, 13901-13917 (1995).

${ }^{20}$ J. Lacasce, "Statistics from Lagrangian observations," Prog. Oceanogr. 77, 1-29 (2008).
${ }^{21}$ R. Lumpkin and S. Elipot, "Surface drifter pair spreading in the North Atlantic," J. Geophys. Res. 115, C12017, doi:10.1029/2010JC006338 (2010).

${ }^{22}$ E. Ser-Giacomi, R. Vasile, E. Hernández-García, and C. López, "Most probable paths in temporal weighted networks: An application to ocean transport," Phys. Rev. E 92, 012818 (2015).

${ }^{23}$ M. Dellnitz, M. Hessel-von Molo, P. Metzner, R. Preis, and C. Schütte, "Graph algorithms for dynamical systems," in Analysis, Modeling and Simulation of Multiscale Problems, edited by A. Mielke (Springer-Verlag, Heidelberg, 2006), pp. 619-645.

${ }^{24} \mathrm{~N}$. Santitissadeekorn and E. Bollt, "Identifying stochastic basin hopping by partitioning with graph modularity," Physica D 231, 95-107 (2007).

${ }^{25}$ V. Rossi, E. Ser-Giacomi, C. López, and E. Hernández-García, "Hydrodynamic provinces and oceanic connectivity from a transport network help designing marine reserves," Geophys. Res. Lett. 41, 2883-2891, doi:10.1002/2014GL059540 (2014).

${ }^{26}$ E. Ser-Giacomi, V. Rossi, C. López, and E. Hernández-García, "Flow networks: A characterization of geophysical fluid transport," Chaos 25, 036404 (2015).

${ }^{27}$ P. Metzner, C. Schütte, and E. Vanden-Eijnden, "Transition path theory for Markov jump processes," Multiscale Model. Simul. 7, 1192-1219 (2009).

${ }^{28} \mathrm{H}$. Kim and R. Anderson, "Temporal node centrality in complex networks," Phys. Rev. E 85, 026107 (2012).

${ }^{29} \mathrm{G}$. Froyland, "An analytic framework for identifying finite-time coherent sets in time-dependent dynamical systems," Physica D 250, 1-19 (2013).

${ }^{30}$ E. W. Dijkstra, "A note on two problems in connexion with graphs," Numer. Math. 1, 269-271 (1959).

${ }^{31}$ H. H. Lentz, T. Selhorst, and I. M. Sokolov, "Unfolding accessibility provides a macroscopic approach to temporal networks," Phys. Rev. Lett. 110, 118701 (2013).

${ }^{32}$ M. Matsueda, "Predictability of Euro-Russian blocking in summer of 2010," Geophys. Res. Lett. 38, L06801, doi:10.1029/2010GL046557 (2011).

${ }^{33}$ E. Black, M. Blackburn, G. Harrison, B. Hoskins, and J. Methven, "Factors contributing to the summer 2003 European heatwave," Weather 59, 217-223 (2004).

${ }^{34}$ S. Saha and coauthors, "The NCEP Climate Forecast System Reanalysis," Bull. Am. Meteor. Soc. 91(8), 1015-1057 (2010).

${ }^{35}$ A. Stohl, C. Forster, A. Frank, P. Seibert, and G. Wotawa, "Technical note: The Lagrangian particle dispersion model FLEXPART version 6.2," Atmos. Chem. Phys. 5, 2461-2474 (2005).

${ }^{36}$ A. Stohl, H. Sodemann, S. Eckhardt, A. Frank, P. Seibert, and G. Wotawa, "The Lagrangian particle dispersion model FLEXPART version 8.2," FLEXPART User Guide (2011), available from http://flexpart.eu/downloads/26.

${ }^{37}$ S. C. Shadden, F. Lekien, and J. E. Marsden, "Definition and properties of Lagrangian coherent structures from finite-time Lyapunov exponents in two-dimensional aperiodic flows," Physica D 212, 271-304 (2005).

${ }^{38}$ M. Farazmand and G. Haller, "Computing Lagrangian coherent structures from their variational theory," Chaos 22, 013128 (2012). 